\title{
Sex differences in emotional insight after traumatic brain injury
}

\author{
Dawn Neumann, PhD, FACRM (Corresponding author) \\ Associate Professor, Indiana University School of Medicine, \\ Department of Physical Medicine and Rehabilitation \\ Rehabilitation Hospital of Indiana \\ 4141 Shore Drive \\ Indianapolis, IN 46254 \\ Email:dmneuman@iupui.edu \\ Phone: 317-329-2188 \\ Barbra Zupan, PhD \\ Associate Professor, Central Queensland University, School of Health, Medical, and \\ Applied Sciences, Department of Exercise and Health Sciences \\ Rockhampton, QLD, Australia 4702
}

\section{*Study was performed at authors' prior institutions:}

Neumann: Carolinas Rehabilitation in the Physical Medicine and Rehabilitation

Zupan: Brock University, Department of Applied Linguistics.

Disclosure: Dawn Neumann was one of the co-creators of a publicly available electronic application that was designed to aid in treating certain aspects of alexithymia. Dr. Neumann does not receive any direct royalties from this App. 
Sex differences and emotional insight

\title{
Sex differences in emotional insight after traumatic brain injury
}

\author{
Dawn Neumann, PhD, FACRM (Corresponding author) \\ Associate Professor, Indiana University School of Medicine, \\ Department of Physical Medicine and Rehabilitation \\ Rehabilitation Hospital of Indiana \\ 4141 Shore Drive \\ Indianapolis, IN 46254 \\ Email: dmneuman@iupui.edu \\ Phone: 317-329-2188 \\ Barbra Zupan, PhD \\ Associate Professor, Central Queensland University, School of Health, Medical, and \\ Applied Sciences, Department of Exercise and Health Sciences \\ Rockhampton, QLD, Australia 4702
}

`Study was performed at authors' prior institutions:

Neumann: Carolinas Rehabilitation in the Physical Medicine and Rehabilitation

Zupan: Brock University, Department of Applied Linguistics. 
Sex differences and emotional insight

\section{$1 \quad$ ABSTRACT}

2 Objective: To compare sex differences in alexithymia (poor emotional processing) in

3 males and females with traumatic brain injury (TBI) and uninjured controls.

4 Setting: TBI rehabilitation facility in the USA and a University in Canada.

5 Participants: Sixty adults with moderate to severe TBI (62\% males) and 60 uninjured

6 controls (63\% males).

7 Design: Cross-sectional.

8 Main Measures: Toronto Alexithymia Scale-20 (TAS-20).

9 Results: Uninjured males had significantly higher (worse) alexithymia scores than

10 uninjured female participants on the TAS-20 $(p=.007)$, whereas, no sex differences were

11 found in the TBI group ( $p=.698)$. Males and females with TBI had significantly higher

12 alexithymia compared to uninjured same-sex controls (both $p s<.001$ ). The prevalence of participants with scores exceeding alexithymia sex-based norms for males and females with TBI was $37.8 \%$ and $47.8 \%$ respectively, compared to $7.9 \%$ and $0 \%$ for male and females without TBI.

Conclusions: Contrary to the majority of findings in the general population, males with TBI were not more alexthymic than their female counterparts with TBI. Both males and females with TBI have more severe alexithymia than their uninjured same-sex peers, and moreover, both are equally at risk for elevated alexithymia compared to norms. Alexithymia should be evaluated and treated after TBI regardless of patient sex. Key Words: emotions, brain injury, alexithymia, affective symptoms, sex differences 
Sex differences and emotional insight

24 Abbreviations:

25 DDF: Difficulty Describing Feelings

26 DIF: Difficulty Identifying Feelings

27 EOT: Externally Oriented Thinking

28 ES: Effect Size

29 GCS: Glasgow Coma Score

30 LOC : Loss of Consciousness

31 PTA : Posttraumatic amnesia

32 SD: Standard Deviation

33 TAS-20: Toronto Alexithymia Scale-20

34 TBI: Traumatic Brain Injury

35

36

37

38

39

40

41

42

43

44

45

46 
Sex differences and emotional insight

Processing emotions is fundamental to psychosocial functioning and overall wellbeing. Alexithymia is an emotional processing deficit that can interfere with the ability to access, recognize, label, differentiate, express, and think about emotions. ${ }^{1-3}$ One common etiology of alexithymia is structural brain damage, which is believed to disrupt areas of the brain involved in processing emotions. ${ }^{4}$ This is referred to as "organic alexithymia" and includes traumatic brain injury (TBI). Non-organic etiologies of alexithymia include childhood or adult psychological trauma, being raised in a culture that discouraged emotional expression, or medical illnesses/ disorders. ${ }^{4}$

Studies show that alexithymia is more common in people with TBI $(31.5 \%$ to $63.9 \%$ ) compared to people without TBI (3.3\% to $15.4 \%)^{1,3,5-7}$ Research in participants with TBI shows that alexithymia is linked to poorer emotional functioning (i.e., anxiety, depression, aggression), unhealthy coping (i.e., avoidant coping, suicide ideation), and reduced quality of life. ${ }^{1-14}$ Given the high rates of alexithymia after TBI and its associations with a variety of negative outcomes, it is important to identify characteristics that might increase risk for alexithymic deficits.

In the general population, one characteristic that has been extensively explored in relation to alexithymia is sex. Findings typically indicate males are more alexithymic than females. ${ }^{8-10}$ A meta-analysis of 41 studies (32 nonclinical and 9 clinical populations ${ }^{8}$ revealed that on average, alexithymia was significantly higher in men than women participants (effect size [E.S] nonclinical sample=.234; clinical sample $\mathrm{ES}=.163) .{ }^{8}$ The Toronto Alexithymia Scale (TAS-20) was used as the outcome measure in $85 \%$ of these studies.). ${ }^{2,11,12}$ This sex discrepancy reported in the general population is most commonly explained by the "Normative Male Hypothesis". 8,10,13-15 This theory 
Sex differences and emotional insight

70 focuses on the effects of gender socialization, postulating that during childhood, men

71 are more often than women discouraged to feel, show, and communicate their emotions, which increases their risk for alexithymia. The authors expected this genderbased theory would only apply to a subset of male participants, thus they anticipated the small effect size found for sex differences in their meta-analysis.

To our knowledge, no study to-date has compared alexithymia differences between males and females with TBI. There was, however, one study conducted in Finland that examined alexithymia in males and females with mild to severe TBI in comparison to uninjured peer controls of the same sex, but not to each other. ${ }^{16}$ That study reported that males with TBI had significantly higher (worse) alexithymia scores than males without TBI, whereas alexithymia scores in females with TBI were similar to their uninjured counterparts. This finding would suggest that women with TBI may not experience alexithymic deficits. However, it was an isolated study that included a sample in which there were twice as many men compared to women. Thus, results for the women $(n=18)$ may have been underpowered. There is a clear need for more research to further explore and potentially replicate this finding. women within the TBI population. Since alexithymia in persons with $\mathrm{TBI}$ is more likely to be the result of the neurological injury (as opposed to socialization), the typical sex differences observed in the general population may not apply to this population. The primary objective of the current study was to compare alexithymia between male and

91 female participants within TBI and non-TBI samples. The secondary objective was to examine how alexithymia in males and females with TBI differs from their uninjured 
Sex differences and emotional insight

counterparts. Although a similar comparison has been explored in another study (described above $)^{16}$, it is unknown if their findings would generalize to a sample that differed in injury severity and geographical location. Within these two study objectives, the prevalence of alexithymia problems will also be explored. However, the traditional TAS-20 cut-off score for severe alexithymia (>60) will not be used for two main reasons. The $>60$ cut-off was a preliminary recommendation based on a small sample of patients referred to a behavioral medicine clinic (14 males, 25 females). ${ }^{11}$ Second, that recommendation applies the same cut-off score for men and women, despite the fact there are sex-based differences in the norms reported for this measure. To overcome these limitations, alexithymia prevalence in this paper will reflect the proportion of participants with alexithymia scores that exceed sex-based norms provided for the TAS20.

\section{METHODS}

\section{Study Design and Setting.}

This cross-sectional study was performed at a rehabilitation hospital in North Carolina, USA and a University in Ontario, Canada. Each site received approval from their ethics committees and all participants provided informed consent before participation. Participants with TBI were recruited via letters and flyers distributed to current and former patients at partnering brain injury facilities, and to local TBI support groups. Peer controls were recruited through flyer advertisements posted at universities and other various community establishments.

This study was conducted as part of a larger research project on emotional 
Sex differences and emotional insight

115 processing after TBI, which resulted in publications that are distinct from the focus and

116 findings of the current manuscript. ${ }^{7,17-19}$

\section{Participants}

This study included 60 participants with TBI (62\% male) and 60 uninjured controls (63\% male) who were frequency matched for age and sex. Participants with TBI met at least one of the Mayo Classification criteria ${ }^{20}$ for moderate to severe TBI

121 (i.e., Glasgow Coma Scale score $<13$ at the time of injury; posttraumatic amnesia $\geq 24$

122 hours; or loss of consciousness for $\geq 30$ minutes). Uninjured controls were excluded for

123 any history of TBI regardless of severity. All participants were excluded for

124 developmental disorders that were affective in nature (e.g., Autism Spectrum Disorder), non-traumatic neurological disorder (e.g., stroke), major psychiatric disorder, and uncorrected vision or hearing impairments that would impede task participation. years of age and had 14-15 years of education. Although the sample was predominantly white, $17.4 \%(n=4)$ of females with TBI were Black/ African American. The majority of participants with TBI were single. For uninjured controls, the majority of females were

131 married, and the males were evenly split between married and single. Participants with

132 TBI were predominantly injured from a motor vehicle accident, and on average, were

133 12-14 post-injury (range: 6 months-37 years). Median loss of consciousness was 21

134 days and 14 days for males and females, respectively. More detail regarding

135 demographics and injury characteristics (TBI only) are presented in Table 1. Power analyses were based on the primary objectives of the larger study, which 
Sex differences and emotional insight

138 Nonverbal Accuracy 2-Adult Faces. ${ }^{21}$ The sample size calculation indicated that 120

139 participants were sufficient to detect medium effect sizes, with $80 \%$ power for

140 independent sample $t$-tests using two tails. Although affect recognition is not the same

141 as alexithymia, they are related constructs. ${ }^{7}$

142

143

144

145

146

147

148

149

150

151

152
[Insert Table 1]

\section{Measures}

Toronto Alexithymia Scale-20 (TAS-20). ${ }^{2,11,12}$ The TAS-20 is comprised of 20 statements that evaluate three alexithymia components: Difficulty Identifying Feelings (DIF); Difficulty Describing Feelings (DDF); and Externally Oriented Thinking (EOT). Participants rate agreement using a Likert scale from 1 (strongly disagree) to 5 (strongly agree). The total TAS-20 scale ranges from 20-100 points. Higher scores indicate a greater degree of alexithymia. Based on TAS-20 norms, average male scores are 47.30 (S.D.=11.32) and average female scores are 44.15 (S.D.=11.19).

\section{Data Analyses}

Two-tailed independent samples $t$-tests were conducted to examine between group differences on demographic variables. A Chi-square test was used to examine if there was a significant difference in the number of males and females in the TBI and uninjured control groups. The relationship of alexithymia outcomes with demographic and injury-related variables were calculated with Pearson correlations. A series of twotailed independent samples $t$-tests were conducted to compare mean alexithymia sex differences within the TBI and uninjured control groups (TAS-20 total and 3 subscales) 
Sex differences and emotional insight

$160(\alpha=.0125)$, as well as for between group comparisons of males with and without TBI

161 and females with and without TBI $(\alpha=.0125)$. Additionally, counts and percentages

162 were calculated to indicate prevalence of participants who met the criteria of having a

163 score greater than one standard deviation above the sex-based norms. This equated to

164 a score of $\geq 59$ for males and $\geq 56$ for females. The relationship between alexithymia

165 classifications with group and sex were explored using Fisher's Exact tests due to Chi-

166 Square violations. SPSS Version 25 was used for all calculations.

167 Results

168 Demographics and Injury Related Variables

169 The TBI and uninjured control groups did not significantly differ in age

$170(t=.146, p=.884), \operatorname{sex}\left(X^{2}=.036, p=.850\right)$, or race $\left(X^{2}=3.748, p=.290\right)$, but did differ by

171 marital status $\left(X^{2}=17.13, p=.002\right)$. Peer controls had significantly more years of

172 education than participants with TBI $(t=-3.06, p=.003)$. Alexithymia was not significantly

173 correlated with age (TBI: $r=-.139, p=.291$; uninjured controls: $r=.037, p=.777)$, race (TBI:

174 rho=-.171, $p=.193$ : HC: $r h o=.061, p=.642$ ), or study site (TBI: $r h o=-.093, p=.482$;

175 uninjured controls: $r h o=.029, p=.823)$ for either group, nor was it correlated with time

176 post-injury $(r=.049, p=.711)$ or PTA $(r=-.053, p=.775)$ for participants with TBI. However,

177 the TAS-20 was significantly correlated with TBI years of education $(r=-.395, p=.002)$

178 and LOC $(r=.335, p=.035)$. Since significant correlations were relatively weak and

179 because this pilot study was not powered to control for these variables, the following

180 analyses do not factor in these variables. This is addressed in more detail in our

181 discussion of limitations. 
Sex differences and emotional insight

Demographics were also examined for sex differences within each group. Males

183

184

and females within the TBI sample did not significantly differ in age $(t=-.181, p=.857)$, education ( $t=.647, p=.521)$, or race $\left(X^{2}=2.960, p=.228\right)$. Similarly, uninjured males and uninjured females did not differ in age $(t=-.776, p=.441)$, education $(t=.931, p=.357)$, or race $\left(X^{2}=.610, p=.737\right)$. Male and female participants with TBI did not significantly differ in time-post injury ( $t=.910, p=.367)$, PTA $(t=.-228, p=.821)$, or LOC $(t=.720 p=.476)$.

Comparisons of alexithymia in males versus females

Statistical values are reported in Table 2 and the proportion of participants with above average alexithymia are shown in Figure 1. Within the TBI sample, mean alexithymia scores for males and females did not significantly differ from one another in the overall TAS-20 score, nor on any of the TAS-20 subconstructs. Effect sizes were also small. In contrast, uninjured male participants had significantly higher scores compared to uninjured female participants on TAS-20 total, DDF, and EOT, with large effect sizes. DIF did not differ between uninjured male and female participants. Fisher's Exact tests indicated that the proportion of males and females who had above average alexithymia did not statistically differ for the TBI group ( $p=.591)$ or the Uninjured controls group $(p=.292)$.

Comparisons of Alexithymia in Participants with TBI versus Uninjured Controls by Sex Compared to uninjured males, male participants with TBI had significantly higher (worse) total alexithymia scores, as well as DIF and DDF subconstructs, but not on EOT. Compared to uninjured female participants, female participants with TBI had significantly higher alexithymia overall and significantly higher scores on all three alexithymia subconstructs. Aside from EOT for males, all effect sizes were large. The 
Sex differences and emotional insight

205

206

207

208

209

210

212

proportion of individuals who had greater than average alexithymia was significantly larger for males and females with TBI ( $p=.002$ and $p<.001$, respectively) compared to their same-sex uninjured peers.

[Insert Table 2 and Figure 1]

\section{Discussion}

Alexithymia is common after TBI and frequently associated with negative psychosocial outcomes. . $^{3,6,7,22-24}$ This is the first study to examine sex differences in alexithymia within a TBI population, and the first to use sex-based norms for identifying alexithymia in a clinical population. Findings from this study revealed that a similarly large proportion of males and females with TBI had alexithymia scores that exceeded sex-based norms (37.8\% to $47.8 \%$, respectively). Further, men and women with TBI had similar alexithymia scores, on average. This suggests alexithymia is a problem for both men and women with TBI. This contrasted findings for the uninjured control group. While proportions of alexithymia in men and women without TBI did not differ $(7.9 \%$ and $0 \%$, respectively), TAS-20 scores for uninjured males were significantly higher than uninjured females for all components but DIF. The sex differences found in our uninjured controls are consistent with prior studies in the general population. ${ }^{825-2831} \mathrm{We}$ suspect that our TBI group did not conform to the typical alexithymia sex differences due to the different etiology of alexithymia in this population (i.e., neurological damage versus gender socialization/ "Normative Male Hypothesis"). ${ }^{9,10,13-15}$ Even if the Normative Male Hypothesis principle was relevant to some participants with TBI prior to their injury, the neurological insult likely overshadows the influence of socialization on 
Sex differences and emotional insight

alexithymia, and thereby diminishes the typical gender-driven differences observed in the general population.

Alexithymia differences between males and females with TBI were also compared to uninjured controls of the same sex. Compared to their uninjured counterparts, male and female participants with TBI had significantly higher alexithymia scores for overall alexithymia and all subconstructs, except EOT, where males with and without TBI did not differ. This finding regarding EOT may suggest that regardless of injury, males are less likely to focus on inner emotional states, as opposed to external factors. Moreover, our results further indicated that a greater proportion of male and female participants with TBI had elevated alexithymia compared to uninjured controls (males: $37.8 \%$ vs $7.9 \%$; females: $47.8 \%$ vs $0 \%$, respectively). These findings contradict results from the Finland study described earlier, ${ }^{16}$ which found that females with TBI did not differ from their uninjured peers, neither by means scores or prevalence of alexithymia $\cdot{ }^{16}$ It is possible our findings differ from the Finland study due to cultural or injury severity differences (we did not include participants with mild TBI). Their female participants may have had milder injuries leading to less of an impact on alexithymia traits. Our females with TBI had higher mean alexithymia scores than theirs (53.39 vs 47.4, respectively). Additionally, the Finland study used the "high" alexithymia cut-off (>60) to determine prevalence of alexithymia for all participants, whereas our study used sex-based norms which may partially explain the discrepancy in identification of women with alexithymia problems between our study (47.8\%) and theirs (22\%). Since women have lower alexithymia norms than men, reaching a universal cut-off would require a bigger deviation from their own norms, than it would for men, to reach "high 
Sex differences and emotional insight

251 alexithymia" levels. Consequently, it is not a fair method for conducting sex 252 comparisons.

253 Research on sex differences after TBI has become a growing focus of interest in 254 TBI outcomes, including other aspects of social cognition. ${ }^{3,29-31}$ It is widely 255 acknowledged that after a TBI, social cognition is frequently impaired ${ }^{32-34}$ and these 256 deficits are associated with worse social outcomes. ${ }^{33}$ The most relevant to alexithymia, 257 is the research on empathy. Past research shows an association between alexithymia 258 and empathy in the TBI population. ${ }^{3,7}$ Consistent with our alexithymia findings, a study 259 examining sex differences in empathy ${ }^{31}$ found that mean empathy scores did not differ 260 between men and women with TBI but a larger proportion of women with TBI (44\%) fell 261 below emotional empathy norms than men with TBI (17\%). ${ }^{31}$ Although in our study the 262 proportions did not differ statistically, the percentage of females who had alexithymia 263 scores that exceeded the norms was larger than that for males (47.8\% vs $37.8 \%)$. Not 264 all sex difference outcomes in the social cognition arena are consistent with our 265 alexithymia findings. ${ }^{3,29,30}$ For example, another study on empathy found men with TBI 266 were more impaired than females. ${ }^{3}$ While it is suspected that cultural differences and 267 the use of different empathy measures could potentially account for these conflicting 268 results, it is evident that more work is needed to expand knowledge and resolve the 269 inconsistency in findings regarding sex differences in social cognition after TBI.

$270 \quad$ Findings from this study should convince researchers and rehabilitation 271 professionals that it is important to take sex and gender-based differences into 272 consideration when working with individuals with TBI to address alexithymia and related 273 social cognition deficits. Clinicians working with people with TBI should evaluate 
Sex differences and emotional insight

274 alexithymia and other emotional processing functions in their patients regardless of sex,

275 and be cognizant of the potential psychosocial impact that deficits in these areas may

276 have on their women patients. Gender expectations for women presume women are

277 emotional, empathic individuals. However, emotional experiences or expressions are

278 often blunted with alexithymia, and related to lower empathy, and are thus discrepant

279 with these expectations preventing females with alexithymia from fitting this

280 stereotypical mold. For men, society often expects them to be less emotionally

281 expressive. Thus, an alexithymic deficit may be more detrimental to women with a TBI

282 than men. It may be helpful for clinicians to determine how much their patients who

283 have alexithymia relate to gender norms relevant to emotional expression, and seek

284 information about family expectations as well. The more discrepancy there is, the more

285 important it will be to educate the patient and families about potential changes

286 associated with alexithymia in how they experience and/ or express their emotions. This

287 process could help to prioritize rehabilitation goals. Early evidence suggests alexithymia

288 is treatable after TBI. ${ }^{6}$ Moreover, targeted treatment can potentially reduce related

289 emotion dysregulation deficits. ${ }^{6}$

290 Limitations and Future Directions

The sample size was driven by a related study objective on emotion perception

292 and was not specifically powered to examine within group alexithymia sex differences

293 nor to control for potential covariates (e.g., years of education). As is typical with

294 preliminary studies with modest sample sizes, results should be interpreted with caution

295 and used to determine appropriate sample sizes needed for future research. Future

296 studies should explore contributions of potential covariates to alexithymia outcomes, 
Sex differences and emotional insight

297

such as education, executive functioning, coping mechanisms, depression, anxiety, and posttraumatic stress disorder (PTSD), which may also vary by sex. Although this study was not heavily unbalanced with regards to sex (60\% male), future research focused on sex differences should strive for equal distribution.

It is also a limitation that we did not collect information about participants' gender identity. While sex and gender are interrelated, there are important distinctions. Sex is primarily defined by biological factors, whereas gender is tied to characteristics that society deems to be more or less stereotypically male or female. Participants may have identified with a gender different from their biological sex, which could have impacted study outcomes. For instance, some females with TBI could possibly have identified with more male-like characteristics, which could have eradicated the sex differences. Future work should collect both sex and gender-related data to better characterize the sample.

Another study limitation is that there are mixed reports in the literature regarding the psychometric properties of the TAS-20, and it has been recommended to be administered in conjunction with other measures. ${ }^{2,11,26,27,35,36}$ To the authors' knowledge, the closest objective measure is the Levels of Emotional Awareness Scale $\left(\right.$ LEAS) ${ }^{37}$, a time-consuming assessment that is not a direct measure of alexithymia nor one that addresses all of the alexithymia constructs. Despite its potential limitation, the TAS-20 was largely selected due to being the most widely used instrument for measuring alexithymia, which facilitates comparison to other studies. Further, it is the only measure specific to alexithymia that has been used in the TBI population. ${ }^{3,5-7,16,22-}$ 24,38-41 The TAS-20 includes statements that people with severe TBI can evaluate as 
Sex differences and emotional insight

characteristic of themselves or not. Future studies could use the LEAS as an objective measure to complement the TAS-20. A final limitation is that it is unknown if the alexithymia identified in the participants with TBI was due strictly to neurological damage or if it was present prior to their injury (i.e., primary and/or secondary alexithymia). Unfortunately, current measures do not have the capacity to make this distinction.

\section{Conclusions}

Study findings suggest that a similarly large proportion of males and females with TBI exceed the alexithymia norms reported for their sex, and in general, have a similar degree of alexithymia. This is in contrast to the general population which typically shows males are more alexithymic. Etiological differences (i.e., organic alexithymia) may be a possible explanation for this difference. Rehabilitation professionals should be aware of these potential emotional deficits in their patients for males and females alike, and should evaluate, educate, and treat accordingly.

\section{Conflicts of Interest/ Grant Funding:}

Dr. Dawn Neumann contributed to the development of an electronic application that is designed to aid in the treatment of alexithymia. She does not receive royalties for the App, but Indiana University does.
Dr. Barbra Zupan has no conflicts of interest.

Source of funding: This work was supported by Cannon Research Center at Carolinas Rehabilitation in Charlotte, North Carolina, and the Humanities Research Institute at Brock University.

Related Presentations: 
Sex differences and emotional insight

Neumann, D., and Zupan, D. Processing Self-emotions after Brain Injury: Women May Have More to Lose than Men. International Brain Injury Association $13^{\text {th }}$ World Congress on Brain Injury, Toronto Canada, March 13-16, 2019.

\section{References}

1. Wood RL, Williams C. Neuropsychological correlates of organic alexithymia. Journal of the International Neuropsychological Society. 2007;13(03):471-479.

2. Bagby RM, Parker JDA, Taylor GJ. The 20-Item Toronto-Alexithymia-Scale .1. Item Selection and Cross-Validation of the Factor Structure. Journal of Psychosomatic Research. 1994;38(1):23-32.

3. Williams C, Wood RL. Alexithymia and emotional empathy following traumatic brain injury. Journal of Clinical and Experimental Neuropsychology. 2010;32(3):259-267.

4. Messina A, Beadle J, Paradiso S. Towards a classification of alexithymia: primary, secondary and organic. Journal of Psychopathology. 2014;20:38-49.

5. Henry J, Phillips L, Crawford J, Theorodou G, Summers F. Cognitive and psychosocial correlates of alexithymia following traumatic brain injury. Neuropsychologia. 2006;44:62-72.

6. Neumann D, Malec JF, Hammond FM. Reductions in Alexithymia and Emotion Dysregulation After Training Emotional Self-Awareness Following Traumatic Brain Injury: A Phase I Trial. The Journal of Head Trauma Rehabilitation. 2017;32(5): 286-295. 
Sex differences and emotional insight

7. Neumann D, Zupan B, Malec J, Hammond F. Relationships between alexithymia, affect recognition, and empathy after traumatic brain injury. The Journal of Head Trauma Rehabilitation. 2014;29(1):E18-27.

8. Levant RF, Hall RJ, Williams CM, Hasan NT. Gender differences in alexithymia. Psychology of men \& masculinity. 2009;10(3):190.

9. Fischer AR, Good GE. Men and psychotherapy: An investigation of alexithymia, intimacy, and masculine gender roles. Psychotherapy: Theory, Research, Practice, Training. 1997;34(2):160.

10. Levant RF, Hirsch LS, Celentano E, Cozza TM. The male role: An investigation of contemporary norms. Journal of Mental Health Counseling. 1992.

11. Bagby RM, Taylor GJ, Parker JDA. The 20-Item Toronto-Alexithymia-Scale .2. Convergent, Discriminant, and Concurrent Validity. Journal of Psychosomatic Research. 1994;38(1):33-40.

12. Taylor GJ, Ryan D, Bagby M. Toward the development of a new self-report alexithymia scale. Psychotherapy and psychosomatics. 1985;44(4):191-199.

13. Levant RF, Allen PA, Lien M-C. Alexithymia in men: How and when do emotional processing deficiencies occur? Psychology of Men \& Masculinity. 2014;15(3):324.

14. Levant RF, Good GE, Cook SW, et al. The normative Male Alexithymia Scale: Measurement of a gender-linked syndrome. Psychology of Men \& Masculinity. 2006;7(4):212.

15. Levant RF, Hall RJ, Rankin TJ. Male Role Norms Inventory-Short Form (MRNI-SF): Development, confirmatory factor analytic investigation of structure, and measurement invariance across gender. Journal of counseling psychology. 2013;60(2):228.

16. Koponen S, Taiminen T, Honkalampi K, et al. Alexithymia after traumatic brain injury: its relation to magnetic resonance imaging findings and psychiatric disorders.

Psychosomatic Medicine. 2005;67(5):807-812.

17. Neumann D, Zupan B. Empathic responses to affective film clips following brain injury and the association with emotion recognition accuracy. Archives of physical medicine and rehabilitation. 2019;100(3):458-463.

18. Zupan B, Neumann D. Using emotional and neutral film clips to investigate impaired affect recognition and empathic responses after TBI. Paper presented at: BRAIN INJURY2012.

19. Zupan B, Neumann D. Affect recognition in traumatic brain injury: Responses to unimodal and multimodal media. The Journal of head trauma rehabilitation. 2014;29(4):E1-E12.

20. Malec JF, Brown AW, Leibson CL, et al. The Mayo classification system for traumatic brain injury severity. Journal of Neurotrauma. 2007;24(9):1417-1424.

21. Nowicki S, Duke MP. Individual differences in the nonverbal communication of affect: The Diagnostic Analysis of Nonverbal Accuracy Scale. Journal of Nonverbal Behavior. 1994;18(1):9-35.

22. Williams C, Wood RL. The impact of alexithymia on relationship quality and satisfaction following traumatic brain injury. The Journal of head trauma rehabilitation. 2013;28(5):E21-E30. 
Sex differences and emotional insight

438

439

440

441

442

443

444

445

446

447

448

449

450

451

452

453

454

455

456

457

458

459

460

461

462

463

464

465

466

467

468

469

470

471

472

473

474

475

476

477

478

479

480

23. Wood RL, Doughty C. Alexithymia and avoidance coping following traumatic brain injury. The Journal of head trauma rehabilitation. 2013;28(2):98-105.

24. Neumann D, Malec JF, Hammond FM. The Relations of Self-Reported Aggression to Alexithymia, Depression, and Anxiety After Traumatic Brain Injury. The Journal of Head Trauma Rehabilitation. 2016 doi: 10.1097/HTR.0000000000000261.

25. Lane RD, Sechrest L, Riedel R. Sociodemographic correlates of alexithymia. Comprehensive psychiatry. 1998;39(6):377-385.

26. Parker JD, Michael Bagby R, Taylor GJ, Endler NS, Schmitz P. Factorial validity of the 20item Toronto Alexithymia Scale. European Journal of personality. 1993;7(4):221-232.

27. Parker JD, Taylor GJ, Bagby RM. The 20-Item Toronto Alexithymia Scale: III. Reliability and factorial validity in a community population. Journal of psychosomatic research. 2003;55(3):269-275.

28. Salminen JK, Saarijärvi S, Äärelä E, Toikka T, Kauhanen J. Prevalence of alexithymia and its association with sociodemographic variables in the general population of Finland. Journal of psychosomatic research. 1999;46(1):75-82.

29. Rigon A, Turkstra LS, Mutlu B, Duff MC. Facial-affect recognition deficit as a predictor of different aspects of social-communication impairment in traumatic brain injury. Neuropsychology. 2018;32(4):476.

30. Zupan B, Babbage D, Neumann D, Willer B. Sex differences in emotion recognition and emotional inferencing following severe traumatic brain injury. Brain Impairment. 2017;18(1):36-48.

31. Zupan B, Neumann D, Babbage D, Willer B. Sex-based differences in Affective and Cognitive Empathy Following Severe Traumatic Brain Injury. Neuropsychology. 2018(32):554-563.

32. May M, Milders M, Downey B, et al. Social behavior and impairments in social cognition following traumatic brain injury. Journal of the International Neuropsychological Society. 2017;23(5):400-411.

33. Milders M. Relationship between social cognition and social behaviour following traumatic brain injury. Brain injury. 2019;33(1):62-68.

34. Neumann D, Zupan B, Eberle R. Social Cognition. In: Jonathon M. Silver TWM, and David B. Arciniegas, ed. Textbook of Traumatic Brain Injury. 3rd ed. Washington, D.C.: American Psychiatric Association Publishing; 2019:281-297.

35. Loas $G$, Corcos M, Stephan P, et al. Factorial structure of the 20-item Toronto Alexithymia Scale: confirmatory factorial analyses in nonclinical and clinical samples. Journal of psychosomatic research. 2001;50(5):255-261.

36. Kooiman C, Spinhoven P, Trijsburg R. The assessment of alexithymia: a critical review of the literature and a psychometric study of the Toronto Alexithymia Scale-20. Journal of psychosomatic research. 2002;53(6):1083-1090.

37. Lane RD, Quinlan DM, Schwartz GE, Walker PA, Zeitlin SB. The Levels of Emotional Awareness Scale: a cognitive-developmental measure of emotion. J Pers Assess. 1990;55(1-2):124-134.

38. Katsifaraki M, Wood RL. The impact of alexithymia on burnout amongst relatives of people who suffer from traumatic brain injury. Brain injury. 2014;28(11):1389-1395. 
Sex differences and emotional insight

481

482

483

484

485

486

487

488

489

490

491

492

493

494

495
39. Wood RL, O'hagan G, Williams C, McCabe M, Chadwick N. Anxiety sensitivity and alexithymia as mediators of postconcussion syndrome following mild traumatic brain injury. The Journal of head trauma rehabilitation. 2014;29(1):E9-E17.

40. Wood RL, Williams C, Kalyani T. The impact of alexithymia on somatization after traumatic brain injury. Brain Injury. 2009;23(7-8):649-654.

41. Wood RL, Williams C, Lewis R. Role of alexithymia in suicide ideation after traumatic brain injury. Journal of the International Neuropsychological Society. 2010;16(06):11081114.

\section{Figures Legends:}

Figure 1. Percentage of Participants with Above Average Alexithymia (>1SD above sex-based norms) 


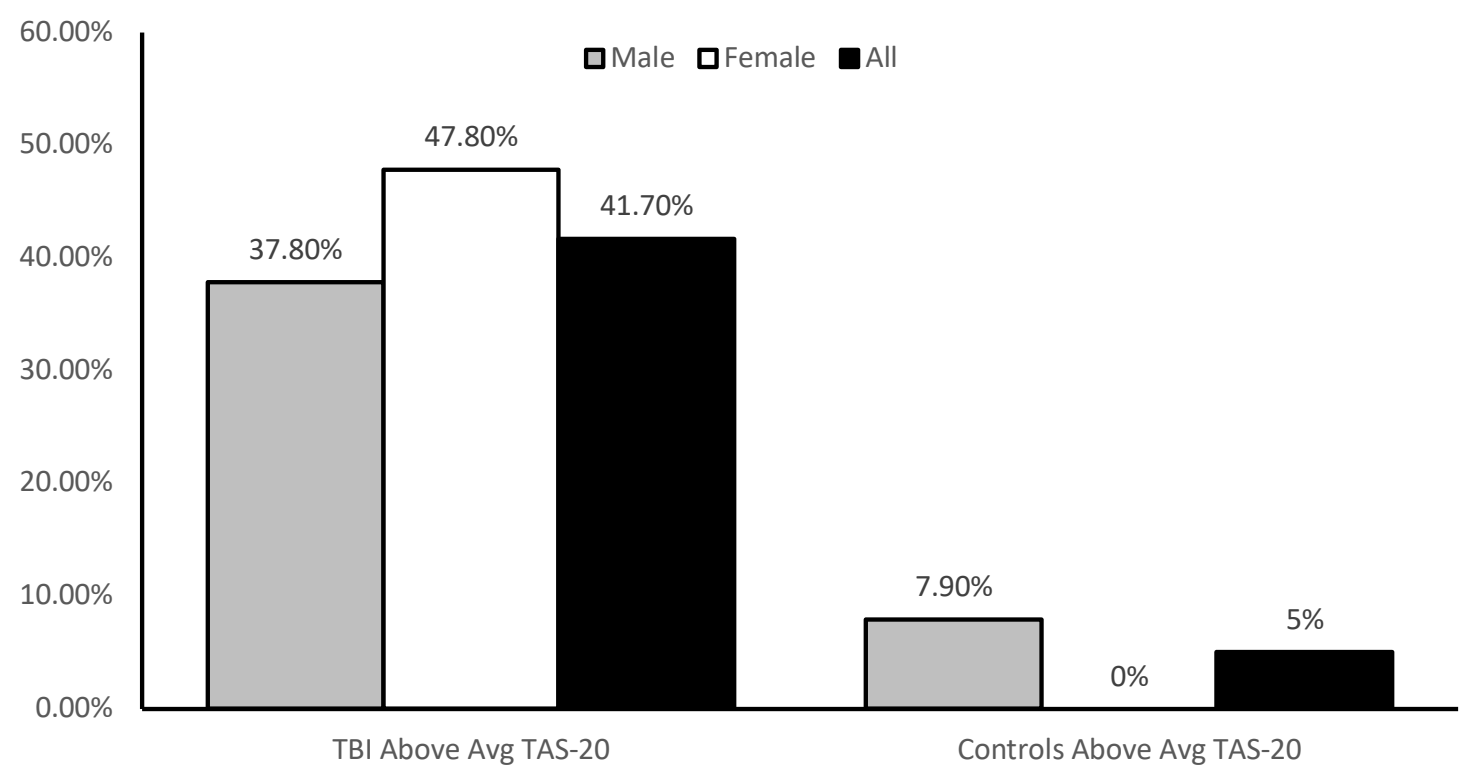

Figure 1

Legend:

Figure 1. Percentage of Participants with Above Average Alexithymia ( $>1 S D$ above sex-based norms) 


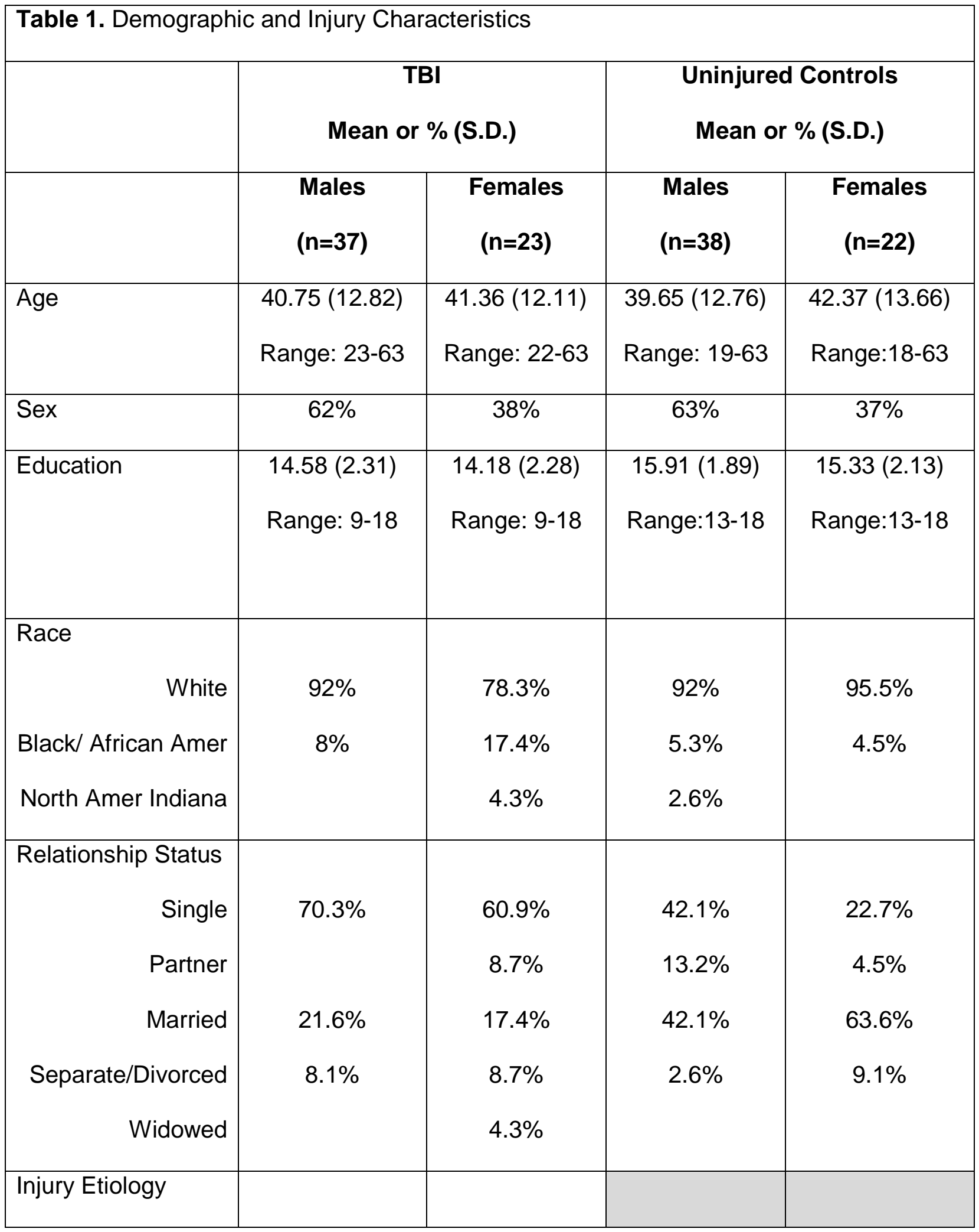




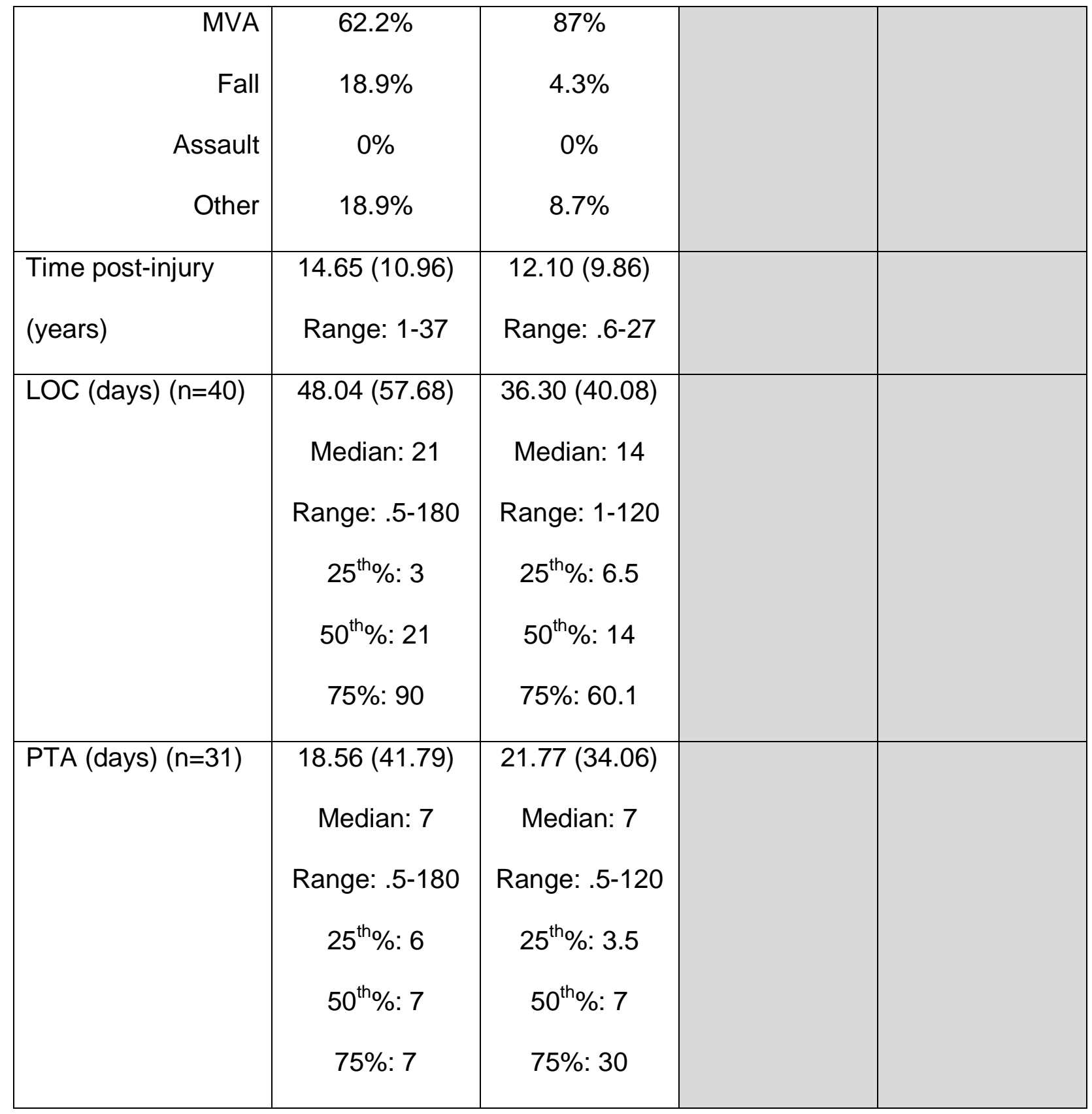

Note: LOC=Loss of consciousness; PTA=Post-traumatic amnesia 



\begin{tabular}{|c|c|c|c|c|c|c|c|c|}
\hline \multicolumn{9}{|c|}{$\begin{array}{l}\text { Table 2: TAS-20 Means, Standard } \\
\text { TBI and Uninjured Controls (Ctrls) }\end{array}$} \\
\hline & \multicolumn{2}{|c|}{$\begin{array}{c}\text { TBI } \\
\text { Mean (S.D.) }\end{array}$} & \multicolumn{2}{|c|}{$\begin{array}{l}\text { Uninjured } \\
\text { Controls } \\
\text { Mean (S.D.) }\end{array}$} & $\begin{array}{c}\text { Male } \\
\text { vs } \\
\text { Female } \\
p \text { value } \\
\text { (ES) }\end{array}$ & $\begin{array}{c}\text { Male } \\
\text { vs } \\
\text { Female } \\
p \text { value } \\
\text { (ES) }\end{array}$ & $\begin{array}{c}\text { TBI } \\
\text { vs } \\
\text { Ctrls } \\
p \text { value } \\
\text { (ES) }\end{array}$ & $\begin{array}{c}\text { TBI } \\
\text { vs } \\
\text { Ctrls } \\
\text { p value } \\
\text { (ES) }\end{array}$ \\
\hline & $\begin{array}{l}\text { Male } \\
(n=37)\end{array}$ & $\begin{array}{l}\text { Female } \\
(n=23)\end{array}$ & $\begin{array}{l}\text { Male } \\
(n=37)\end{array}$ & $\begin{array}{l}\text { Female } \\
(n=38)\end{array}$ & $\begin{array}{c}\text { TBI } \\
(n=22)\end{array}$ & Ctrl & Male & Female \\
\hline $\begin{array}{r}\text { TAS-20 } \\
\text { Total }\end{array}$ & $\begin{array}{l}54.65 \\
(11.53)\end{array}$ & $\begin{array}{r}53.39 \\
(13.09)\end{array}$ & $\begin{array}{r}43.53 \\
(10.42)\end{array}$ & $\begin{array}{l}36.09 \\
(9.06)\end{array}$ & $\begin{array}{l}t=.390 \\
p=.698 \\
(0.10)\end{array}$ & $\begin{array}{c}t=2.791 \\
p=.007 \\
(0.76)\end{array}$ & $\begin{array}{c}t=4.387 \\
p<.001 \\
(1.01)\end{array}$ & $\begin{array}{c}t=5.132 \\
p<.001 \\
(1.54)\end{array}$ \\
\hline DIF & $\begin{array}{l}18.46 \\
(6.34)\end{array}$ & $\begin{array}{l}19.13 \\
(6.69)\end{array}$ & $\begin{array}{l}11.87 \\
(5.24)\end{array}$ & $\begin{array}{l}11.27 \\
(4.82)\end{array}$ & $\begin{array}{l}t=-.390 \\
p=.698 \\
(0.10)\end{array}$ & $\begin{array}{c}t=.436 \\
p=.664 \\
(0.11)\end{array}$ & $\begin{array}{c}t=4.914 \\
p<.001 \\
(1.13)\end{array}$ & $\begin{array}{c}t=4.820 \\
p<.001 \\
(1.35)\end{array}$ \\
\hline DDF & $\begin{array}{l}15.27 \\
(3.75)\end{array}$ & $\begin{array}{l}14.22 \\
(4.27)\end{array}$ & $\begin{array}{l}12.39 \\
(4.19)\end{array}$ & $\begin{array}{l}9.05 \\
(2.8)\end{array}$ & $\begin{array}{l}t=1.00 \\
p=.321 \\
(0.26)\end{array}$ & $\begin{array}{c}t=3.337 \\
p=.001 \\
(0.94)\end{array}$ & $\begin{array}{c}t=3.128 \\
p=.003 \\
(0.72)\end{array}$ & $\begin{array}{c}t=4.820 \\
p<.001 \\
(1.43)\end{array}$ \\
\hline EOT & $\begin{array}{l}20.92 \\
(5.16)\end{array}$ & $\begin{array}{l}20.04 \\
(5.58)\end{array}$ & $\begin{array}{l}19.27 \\
(4.22)\end{array}$ & $\begin{array}{l}15.77 \\
(4.58)\end{array}$ & $\begin{array}{l}t=.619 \\
p=.538 \\
(0.16)\end{array}$ & $\begin{array}{c}t=2.983 \\
p=.004 \\
(0.79)\end{array}$ & $\begin{array}{c}t=1.504 \\
p=.137 \\
(0.35)\end{array}$ & $\begin{array}{c}t=2.800 \\
p=.007 \\
(.84)\end{array}$ \\
\hline
\end{tabular}


